\title{
Health Information Technology and Care Coordination:The Next Big Opportunity for Informatics?
}

\author{
David W. Bates ${ }^{1,2,3}$ \\ 1 Division of General Internal Medicine, Brigham and Women's Hospital, Boston, MA, USA \\ 2 Harvard Medical School, Boston, MA, USA \\ ${ }^{3}$ Department of Health Policy and Management, Harvard School of Public Health, Boston, MA, USA
}

\section{Summary}

The costs of care in the U.S. are very high, in part because canre is relatively uncoordinated. To begin to address this and other issues, health care reform was passed, including the notion of accountable care. Under acountable care arrangements, providers are at risk for the costs of the care they provide to groups of patients. Evaluation of costs has made it clear that a large proportion of these costs are in the post-acute setting, and also that many specific problems such as adverse events and unnecessary readmissions occur following transitions. However, the electronic health records of today do not provide a great deal of assistance with the coordination of care, and even the best organizations have relatively primitive systems with respect to care coordination, even though communication is absolutely central to better coordination of care and health information technology (HIT) is a powerful lever for improving communication. This paper identifies specific gaps in care coordination today, presents a framework for better coordinating care using HIT, then describes how specific technologies can be leveraged. Also discussed are the need to build and test specific interventions to improve HIT-related care coordination tools, and the key policy steps needed to accomplish this.

\section{Keywords}

Health information technology, electronic health records, meaningful use, quality, efficiency, accountable care organization

Yearb Med Inform 2015;10:11-4

http://dx.doi.org/10.15265/IY-2015-020

Published online June 30, 2015
The costs of healthcare in the U.S. are far too high, and yet objective measures show that quality and safety are low, which has resulted in low-value care [1]. The rising costs have led to attempts to reduce care expenditures, notably recently through the passage of the Accountable Care Act. A major target of this legislation was to improve the proportion of the population with health insurance, which it did successfully. It has been criticized, though, for not sufficiently tackling the issue of cost.

Another major goal of the legislation was to enable the establishment of Accountable Care Organizations (or ACOs). The concept of ACO legislation is that it will enable organizations to contract to deliver care for groups of patients for a fixed price. When costs are examined however, one of the emerging key findings is that approximately $70 \%$ of costs occur outside of hospitals. This leads to the conclusion for ACOs to be successful in managing costs they will need to do so across the continuum, including sectors, where they have had little input historically, including long-term, post-acute, and home care, as well as the outpatient setting.

This paper begins by making the assertion and reviews some of the evidence suggesting that care today is largely uncoordinated, then identifies a number of specific gaps, presents a framework for better coordinating care using HIT, then describes how specific technologies can be leveraged and the need to build and test specific interventions, and then finally discusses the policy needs in this area.

\section{Care Today Is Largely Uncoordinated}

The existing evidence suggests that care today in the U.S. is largely uncoordinated even though evidence also suggests that quality improvement strategies within care can improve performance [2,3]. There are a number of indices of this. For example, the average Medicare beneficiary sees many different providers in a given year and network characterist- -lectronic health records (EHRs), in particular, are not yet doing very much to date to improve this situation $[5,6,7]$. While the Health Information Technology for Economic and Clinical Health (HITECH) Act has resulted in rapid adoption of EHRs in most hospitals and also in ambulatory care, nursing homes, post-acute settings, and home care were not eligible for the HITECH incentives. Furthermore, arguably the most important piece of progress that was needed for improving the situation - clinical data exchange-has lagged far behind EHR adoption rates [8].

Medical homes represent an important effort to try to better manage and coordinate care for populations [9]. O'Malley et al explored how electronic records were working in medical homes with respect to care coordination [6]. Her team identified four especially important domains for EHRs in care coordination: 1) reconciling medications, 2) tracking laboratory tests, 3 ) communicating across settings, and 4) mediating care plans between disciplines. Managing referrals, consultations, and 
care transitions were also important. Today's EHRs have substantial deficiencies in all these areas.

Our group did a national evaluation of how well HIT was being used to support care transitions across settings [10]. We expected to find some exemplar organizations which had sophisticated tools for managing these, and did find some organizations which appeared to be performing better than others, but in every instance this was occurring largely with "high touch" human involvement and without leveraging technologies. We did however find that some HIT tools were being used within one healthcare setting to prepare for transitions of care.

\section{Key Gaps}

With respect to medication reconciliation, some good electronic tools for managing medication have been built (Figure 1), but they are not yet in wide use [11]. Furthermore, it will be essential to bring dispensing or "fill" data into these tools in a relatively seamless way, which has not been done except within closed systems.

Similarly, tools to enable the tracking of laboratory tests (Figure 2) have been developed in a few systems [12], but are not yet routinely available. These should enable at least the following functions: they should group results by provider, prioritize them according to degree of abnormality, and make it easy for providers to rapidly generate a message to the patient about the results. In addition, population-level tools are needed to be able to aggregate certain tests results (e.g. abnormal Pap smears and high Prostate Specific Antigen or PSA), so that one individual can be tasked with ensuring that $100 \%$ of these receive appropriate follow-up.

Communicating across care settings represents a major opportunity-clearly, data exchange will help with this - but implementing additional HIT communication tools could have a very big impact. Today, EHRs do not routinely track who is part of the team caring for the patient, or who a patient's specialists may be. Many tools available today also do not show whether or not a given individual is available at work. Not knowing this information leads to many of the "dropped balls" or incomplete tasks in today's systems.

The issue of care plans is also centrally important [13]. Most EHRs today do not include a place to develop, store, and share a care plan, and there is a lack of widespread agreement about what should be included in a care plan. Robust standards in this area would be helpful. Most prior work has come from nursing, but truly multi-disciplinary care plans will be needed if we are to get to a better place in care coordination.

Referrals also represent a major gap. EHRs often enable providers to generate referrals, but they are mostly "open-loop" requests and do not typically include tools to ensure that important referrals actually occur-it is left to the referrer to follow up, which often does not happen. For many referrals, this is not very important, but if a patient has a breast mass for example it is critical to ensure that she receives appropriate follow-up. The next generation of referral tools should also enable "electronic curbsides," - the ability to ask a specialist for advise without sending the patient - as some patients may not need an appointment with a specialist if a question can readily be addressed, reducing costs and overcrowding at specialists. These tools could also help ensure that patients get to the best specialist for their specific problem. Many specialists focus on very narrow areas, and it can be hard for primary care providers to find the best fit for their patients.

Yet another opportunity is improved communication within teams for complex patients-within Medicare populations, only about $5 \%$ of patients account for $50 \%$ of costs [14]. This group of patients requires intensive attention from a team - which will include their primary care provider and associated team members, specialists, likely a care coordinator, and perhaps a behavioral health provider or a pharmacist. This entire group needs to be able to communicate better than they do today-to see the care plan, to message each other, and to have synchronous and asynchronous conversations about goals, and progress or lack thereof. A similar set of issues exists for complex inpatients-with many providers caring for these patients and the norm being highly fragmented communication.

A related issue that organizations have not leveraged is the finding that nearly all patients have what has been called a "care partner," even though they may not have a care-giver [15]. A care partner is someone, who will help a patient when they need assistance, for example after a surgical procedure or colonoscopy, and often are relatives or friends. These care partners

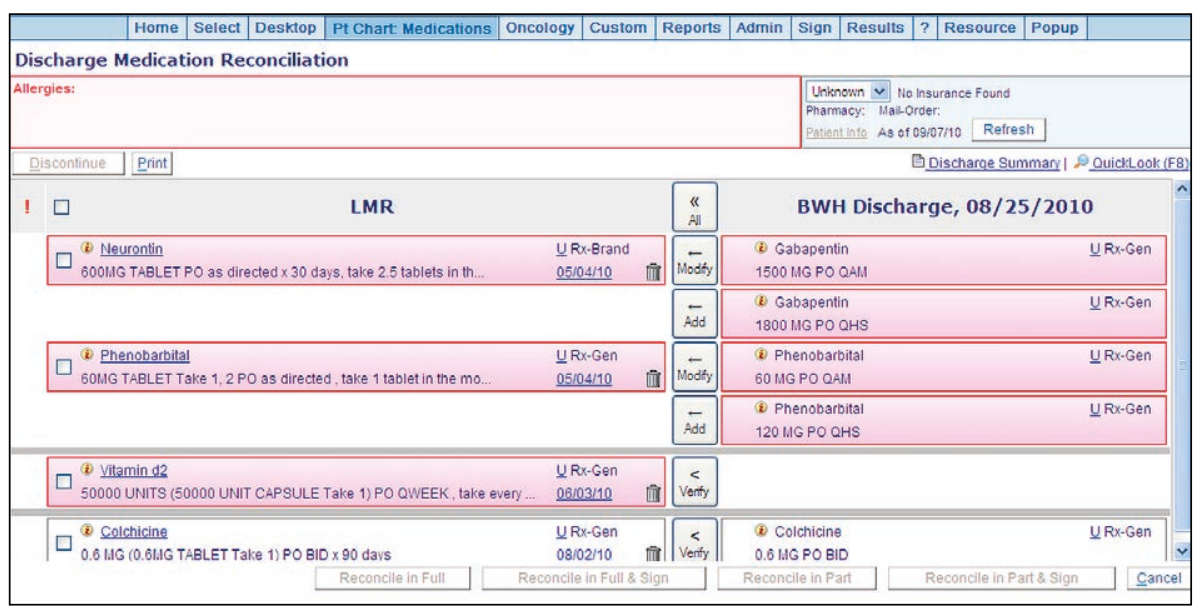

Fig. 1 Medication reconciliation. Providers can see both the discharge medications (on the right) and the pre-admission medications on the left, making it easier to do the reconciliation. 


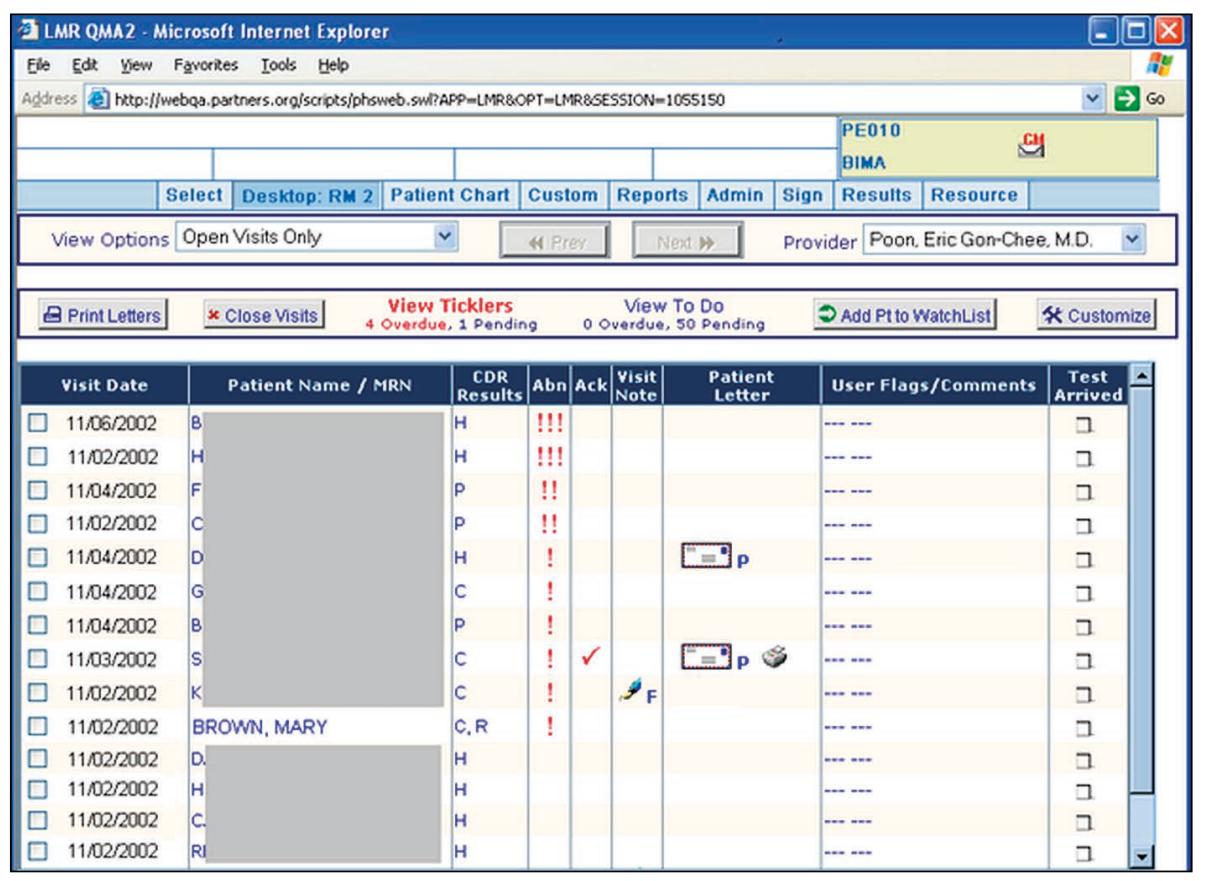

Fig. 2 Tool to enable results management. Puts all a provider's results on one page, prioritizes them according to degree of abnormality, and allows a provider with a click to generate a letter to the patient.

represent a substantial resource, and from the HIT perspective the first step is to begin to track these individuals and to categorize their relationship with the patient. Such care partners may be especially important in helping care for patients with cognitive impairment or behavioral health issues.

Outside the hospital and crossing care settings, the situation is even worse. HITECH covers primarily hospitals and outpatient providers, which has left many gaps in the advancement of EHRs, including long-term care and home care settings. Many of these providers are not yet using EHRs, and the few who are use relatively unsophisticated ones. In one study, both home care providers and primary care providers perceived their biggest need to be able to communicate better with each other. Each group thought that the other group was "in control" of the overall process [16]. If organizations are to manage care across the continuum, they will need much better information regarding events in long-term and home care, and the communication and in particular hand-offs will need to be done more effectively.

\section{A Framework for Better Coordinating Care Using HIT}

One framework for better coordinating care using HIT was recently put forward by Rudin and Bates [17]. We suggested that there were four central categories that providers need to address to perform care coordination- - that they need to be able to identify collaborators, contact collaborators, collaborate, and monitor. Each of these functionalities has major issues today. Most EHRs do not reliably identify a patient's care team, and it can be especially hard to identify community resources. Contacting collaborators is relatively easier, but a major limitation today is that many such contact requests do not include the level of urgency, and the availability or "interruptability" status of collaborators is often unknown. Much more robust tools are needed to actually collaborate, for example the ability to hold secure videoconferences, and to send images. Finally, tools that track provider responsibilities for monitoring ongoing care including safeguards and escalation procedures are needed.

\section{Leveraging Technologies}

One of the great strengths of information technology is that it can enable communication. But much of the technology in use today in healthcare is very old - substantial quantities of information are still faxed, and paging is still in routine use. There are innumerable opportunities to improve communication within care teams and with potential new collaborators, including free text, structured text, videoconferencing, and microblogs, to name just a few. The use of mobile technology in healthcare is also in its infancy.

Many use cases exist where HIT would offer advantages. For example, it should be made easy for providers to set up a secure videoconference with their longitudinal patients. Another example is providers trying to refer a patient to a specialist colleague. Today they usually can't readily find out exactly what type of referrals the specialist may be seeking or what their availability for referrals is.

\section{Interventions}

There is a clear need to build and test interventions to improve care coordination. One example of an intervention which targets this area is a toolkit our group has built for the PROSPECT trial [18], which is attempting to improve outcomes in intensive care and in general care units. The tools built include a patient-centered toolkit, which the patient or care partner can access and which includes information about the plan of care and goals of care visible to the entire care team. In addition, we have developed a microblog function, so that anyone on the team including the patient or the care partner can ask a question, anyone can respond, and everyone in the care team can see what the responses of the others have been [18].

Many other interventions are being tested internationally which include HIT and partly address care coordination. One example is two linked trials to improve care for patients with depression and cardiovascular disease risk in the UK [19]. Another example is the Finnish Geriatric Intervention Study to prevent cognitive impairment and disability [20]. The issues involved vary substantially 
by setting. For example, outside the hospital, access to information about community resources, such as substantial abuse treatment and mental health care, represents a particular challenge. Many such interventions need to be designed and tested, in a variety of settings.

\section{Policy Needs}

The first need in this area is support for research on improving care coordination within the context of electronic health records and medical homes. This should be supported through the Agency for Healthcare Policy and Research and the National Library of Medicine - multiple experiments are needed, especially within the context of medical homes, and some should be done in locations, where health information exchange is occurring, as one of the key needs is better communication. Once robust tools have been developed, the Center for Medicaid and Medicare Innovation (CMMI) could promote their spread. In addition, the Office of the National Coordinator should support further development of standards in this area, especially but not exclusively for care plans. The Continuity of Care Document (CCD) summarizes the clinical information for a patient, and is the main approach being used to exchange information between EHRs. How well it works, though, for care coordination is uncertain. The strengths and weaknesses of the CCD standard should also be explored through this lens-while organizations can increasingly create CCDs, it is unclear about how well they support care coordination needs, and what modifications or extensions might be helpful. Adoption of such tools is likely to be closely linked to broader health care reform initiatives, in particular the implementation of accountable care or other payment approaches that place providers at risk, especially for care outside the walls of the hospital.

\section{Conclusions}

If organizations want to succeed in improving quality and reducing costs, providing better with care coordination is one of the most important keys. However, the electronic health records of today do not yet truly enable care coordination. Even the leading U.S. organizations in care coordination do not yet have robust electronic tools for doing this - making this a key frontier for clinical informatics.

\section{References}

1. Davins K, Stremikis K, Squires D, Schoen C. Mirror, Mirror on the Wall, 2014 Update: How the U.S. Health Care System Compares Internationally. The Commonwealth Fund; 2014.

2. Tricco AC, Antony J, Ivers NM, Ashoor HM, Khan PA, Blondal E, et al. Effectiveness of quality improvement strategies for coordination of care to reduce use of health care services: a systematic review and meta-analysis. CMAJ 2014;186(15):E568-78.

3. Schultz EM, Pineda N, Lonhart J, Davies SM, McDonald KM. A systematic review of the care coordination measurement landscape. BMC Health Serv Res 2013;13:119.

4. Landon BE, Keating NL, Barnett ML, Onnela JP, Paul S, O'Malley AJ, et al. Variation in patient-sharing networks of physicians across the United States. JAMA 2012;308(3):265-73.

5. Mehrotra A, Forrest CB, Lin CY. Dropping the baton: specialty referrals in the United States. Milbank Q 2011;89(1):39-68.

6. O'Malley AS, Grossman JM, Cohen GR, Kemper NM, Pham HH. Are electronic medical records helpful for care coordination? Experiences of physician practices. J Gen Intern Med 2010;25(3):177-85.

7. Nutting PA, Crabtree BF, Miller WL, Stange KC, Stewart E, Jaén C. Transforming physician practices to patient-centered medical homes: lessons from the national demonstration project. Health Aff (Millwood) 2011;30(3):439-45.

8. Adler-Milstein J, Bates DW, Jha AK. A survey of health information exchange organizations in the United States: implications for meaningful use. Ann Intern Med 2011;154(10):666-71.

9. Bates DW, Bitton A. The future of health information technology in the patient-centered medical home. Health Aff(Millwood) 2010;29(4):614-621.
10. Samal L, Dykes PC, Greenberg J, Hasan O, Venkatesh AK, Volk LA, et al. The current capabilities of health information technology to support care transitions. AMIA Annu Symp Proc 2013;2013:1231.

11. Schnipper JL, Hamann C, Ndumele CD, Liang CL, Carty MG, Karson AS, et al. Effect of an electronic medication reconciliation application and process redesign on potential adverse drug events: a cluster-randomized trial. Arch Intern Med 2009;169(8):771-80.

12. Poon E, Wang S, Gandhi T, Bates DW, Kuperman GJ. A Comprehensive Outpatient Results Manager with Decision Support: Design Considerations and Architecture. Proc AMIA Symp; 2002.

13. Dykes PC, Samal L, Donahue M, Greenberg JO, Hurley AC, Hasan O, et al. A patient-centered longitudinal care plan: vision versus reality. J Am Med Inform Assoc 2014;21(6):1082-90.

14. Cohen SB, Yu W. The Concentration and Persistence in the Level of Health Expenditures over Time: Estimates for the U.S. Population, 20082009. Rockville, MD: Agency for Healthcare Research and Quality; 2012.

15. Sarkar U, Bates DW. Care partners and online patient portals. JAMA 2014;311(4):357-8.

16. Fairchild DG, Wilcox CM. Comprehending coordinated comprehensive care: the devil is in the dollars. J Gen Intern Med 2007;22(3):424-5.

17. Rudin RS, Bates DW. Let the left hand know what the right is doing: a vision for care coordination and electronic health records. J Am Med Inform Assoc 2014;21(1):13-6.

18. Dalal AK, Dykes PC, Schnipper JL, Bates DW. Transforming the acute care environment: A webbased patient-centered toolkit. Vol 9: Journal of Hospital Medicine; 2014.

19. Walters P, Barley EA, Mann A, Phillips R, Tylee A. Depression in primary care patients with coronary heart disease: baseline findings from the UPBEAT UK study. PLoS One 2014;9(6):e98342.

20. Ngandu T, Lehtisalo J, Levälahti E, Laatikainen T, Lindström J, Peltonen M, et al. Recruitment and baseline characteristics of participants in the Finnish Geriatric Intervention Study to Prevent Cognitive Impairment and Disability (FINGER)-a randomized controlled lifestyle trial. Int J Environ Res Public Health 2014;11(9):9345-60.

Correspondence to:

David Bates

Division of General Internal Medicine

1620 Tremont St.

Boston, MA 02115

United States of America

E-mail:dbates@partners.org 